DOI: 10.12957/demetra.2018.27515

\title{
Avaliação do perfil nutricional e qualidade de vida após realização de cirurgia bariátrica em um Hospital Público de Goiânia, Brasil
}

\section{Nutritional profile and quality of life assessment after bariatric surgery in a public hospital of Goiania, Brazil}

\author{
Ruanda Pereira Maia' \\ Pauliene Christielly Cardoso da Silva' \\ Amélia Cristina Stival Duarte \\ Raphaela Moiana da Costal \\ ' Secretaria Estadual de Saúde de Goiás, Hospital \\ Alberto Rassi - HGG, Programa de Residência \\ Multiprofissional em Clínica Especializada: \\ Endocrinologia no HGG. Goiânia, GO, Brasil. \\ Correspondência / Correspondence \\ Ruanda Pereira Maia \\ Hospital Alberto Rassi - HGG \\ Av. Anhanguera $n^{0} 6.479$, Setor Oeste, CEP: \\ 74110-010, Goiânia-G0, Brasil. \\ E-mail: ruanda_maia@yahoo.com.br
}

\section{Resumo}

Objetivo: avaliar o perfil nutricional e qualidade de vida de indivíduos submetidos a cirurgia bariátrica em um hospital público de Goiânia. Metodologia: Estudo transversal, realizado entre agosto a novembro de 2016, com 56 pacientes do ambulatório de obesidade com 6, 12 e 18 meses de pós-operatório de bypass gástrico em Y de Roux. Analisaram-se perfil antropométrico, presença de comorbidades, hábitos de vida e qualidade de vida por meio do instrumento BAROS. Resultados: $84 \%$ do sexo feminino, com média de idade de 46 anos $(\mathrm{DP}=9,61)$ e nível de escolaridade predominante ensino fundamental incompleto $(44 \%)$. No período pré-operatório, a média de IMC foi $50,1 \mathrm{~kg} / \mathrm{m}^{2} \pm 8,37$ e a de perda de peso, $8,7 \%$. Após a cirurgia bariátrica, a média do IMC foi $35,7 \mathrm{~kg} / \mathrm{m}^{2} \pm 7,09$. A perda de excesso de peso foi superior a $50 \%$ em $78 \%$ da amostra, e houve altos índices de resolução de distúrbios do sono (94\%), dislipidemias (91\%) e diabetes $(86 \%)$ $(\mathrm{p}<001)$. O consumo de bebida alcóolica foi encontrado em $3,7 \%$ dos indivíduos e $74 \%$ referiram praticar atividade física. A qualidade de vida foi avaliada como melhorada (27\%) e muito melhorada (73\%). Entre os três períodos pós-operatórios e entre os indivíduos que tiveram perda ponderal inferior e superior a $50 \%$, não se constataram diferenças significativas, embora todos tenham relatado melhora nos domínios avaliados. Conclusões: a cirurgia bariátrica tem demonstrado eficácia no auxílio na perda de peso, melhora das comorbidades e da qualidade de vida de indivíduos obesos.

Palavras-chave: Cirurgia Bariátrica. Obesidade. Qualidade de Vida. 


\section{Abstract}

Objective: to evaluate the nutritional profile and quality of life of individuals undergoing bariatric surgery in a public hospital of Goiânia-GO, Brazil. Methodology: cross-sectional study conducted between August and November 2016, with 56 patients at the 6 , 12 and 18-month postoperative period of Roux-en-Y Gastric Bypass. Anthropometric profile, presence of comorbidities, life habits and quality of life were analyzed through the BAROS instrument. Results: $84 \%$ were female, with a mean age of 46 years $(\mathrm{SD}=9.61)$, mostly wuth incomplete primary school level $(44 \%)$. In the preoperative period the mean BMI was $50.1 \mathrm{~kg} /$ $\mathrm{m}^{2} \pm 8.37$ and the weight loss was $8.7 \%$. After bariatric surgery, the mean BMI was $35.7 \mathrm{~kg} / \mathrm{m}^{2} \pm 7.09$. The loss of excess weight was greater than $50 \%$ in $78 \%$ of the sample. High resolution indexex of sleep disorders (94\%), dyslipidemias (91\%) and diabetes $(86 \%)$ ( $\mathrm{p}<0.001)$. Alcohol consumption was found in $3.7 \%$ of the individuals and $74 \%$ reported practicing physical activity. Quality of life was assessed as improved (27\%) and greatly improved (73\%). There were no significant differences between the three postoperative periods and between the individuals who had a weight loss of less than $50 \%$ and over $50 \%$, although all reported improvement in the domains evaluated. Conclusions: Bariatric surgery was effective in in aiding weight loss, improving comorbidities and quality of life in obese individuals.

Keywords: Bariatric Surgery. Obesity. Quality of Life.

\section{Introdução}

A obesidade é uma doença crônica caracterizada pelo acúmulo excessivo de gorduras no organismo, que compromete o estado de saúde e qualidade de vida do indivíduo, além de aumentar o risco de mortalidade e incidência de outras doenças crônicas não transmissíveis (DCNT). De etiologia múltipla, pode ter relação com fatores genéticos, socioculturais, ambientais e/ou comportamentais, e acomete atualmente $52,2 \%$ do total da população brasileira. ${ }^{1-4}$

Segundo a Organização Mundial da Saúde (OMS), o índice de massa corporal (IMC) é um indicador utilizado para classificar o indivíduo conforme seu peso e sua altura. É válido também para estratificar os níveis de sobrepeso e obesidade, de modo que valores de IMC $\geq 30$ e $<35$ $\mathrm{kg} / \mathrm{m}^{2}$ são considerados como Obesidade grau I; $\geq 35$ e $<40 \mathrm{~kg} / \mathrm{m}^{2}$ como Obesidade grau II; e $\geq 40 \mathrm{~kg} / \mathrm{m}^{2}$ como Obesidade grau III ou Obesidade grave. ${ }^{5}$ 
O tratamento da obesidade consiste em intervenções farmacológicas, dietéticas, nos hábitos de vida (estímulo à prática de atividade física) e terapia cognitivo-comportamental. Quando ocorre refratariedade ao tratamento conservador após acompanhamento regular por no mínimo dois anos, a cirurgia bariátrica pode ser indicada para determinadas condições clínicas. ${ }^{2,3} \mathrm{~A}$ técnica mais utilizada é o bypass gástrico com desvio intestinal em Y de Roux (Fobi-Capella), considerada como cirurgia mista de caráter restritivo e disabsortivo, capaz de propiciar perda ponderal de 30 a $40 \%$ do peso total. ${ }^{6}$

A perda de peso sustentada é um dos objetivos da cirurgia bariátrica, pois se espera que as comorbidades associadas à obesidade melhorem ou entrem em remissão, e que se obtenha melhora na qualidade de vida e redução na morbimortalidade. ${ }^{7}$

O conceito de qualidade de vida, por sua vez, possui diferentes abordagens e pode ser compreendido como um ideal de saúde, estilo de vida, percepção pessoal na sua posição de vida e no sistema de valores nos quais se vive, entre outros. ${ }^{8}$ Aspectos do estilo de vida como etilismo, tabagismo ${ }^{9}$ e sedentarismo podem estar associados ao quadro de obesidade, e assim na percepção da qualidade de vida individual. Estudos têm apontado associações entre IMC elevado e menor capacidade de trabalho, quando relacionado às exigências físicas de trabalho em indivíduos

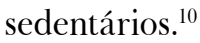

Oria \& Moorehead ${ }^{11}$ elaboraram um protocolo para avaliar a eficiência e o impacto da cirurgia bariátrica na qualidade de vida dos pacientes obesos, denominado Bariatric Analysis and Reporting Outcome System (BAROS). Esse instrumento avalia a perda de excesso de peso, a melhora das comorbidades e cinco aspectos referentes à qualidade de vida, entre eles: autoestima, disposição para atividades físicas, desempenho no trabalho, interação social e prática de atividade sexual. ${ }^{12,13}$

Nesse sentido, tendo em vista que a cirurgia bariátrica é considerada um tratamento eficaz no controle de peso, no prognóstico positivo das comorbidades associadas e também da qualidade de vida de pacientes obesos, o presente estudo teve como objetivo avaliar o perfil nutricional e a qualidade de vida de indivíduos submetidos a cirurgia bariátrica em um hospital público da cidade de Goiânia-GO, caracterizando o perfil antropométrico, hábitos de vida no período pósoperatório e alterações na percepção da qualidade de vida.

\section{Metodologia}

O estudo foi do tipo transversal, de caráter quantitativo, em que foram obtidos dados relacionados a perfil antropométrico, presença de morbidades, hábitos de vida (etilismo, tabagismo e prática de atividade física) e qualidade de vida de adultos submetidos a cirurgia bariátrica, no período de agosto a novembro de 2016 em um hospital público de Goiânia, após terem assinado o Termo de Consentimento Livre Esclarecido (TCLE). 
O cálculo amostral foi realizado com base nos dados do número total de cirurgias realizadas no período de janeiro de 2015 a abril de 2016 (n=77). Foram considerados nível de confiança de $95 \%$ e margem de erro de $10 \%$, totalizando 20 participantes.

A amostra foi composta por indivíduos cadastrados no programa de obesidade e cirurgia bariátrica da instituição. Como condição para a pesquisa, todos os candidatos deveriam ter realizado bypass gástrico em Y de Roux (Fobi-Capella), no período compreendido entre fevereiro de 2015 a maio de 2016. Os integrantes foram classificados em três grupos, conforme o período pós-operatório na data da coleta: (a) Grupo 1: compreendendo os pacientes com seis meses de período pós-operatório (cirurgia realizada de fevereiro a maio de 2016); (b) Grupo 2: indivíduos com 12 meses de pós-operatório (agosto a novembro de 2015); e (c) Grupo 3: pacientes com 18 meses de período pós-operatório (fevereiro a maio de 2015).

Foram incluídos na amostra indivíduos de ambos sexos, com idade igual ou superior a 18 e inferior a 65 anos; com IMC $\geq 35 \mathrm{~kg} / \mathrm{m}^{2}$ associado a comorbidades como diabetes mellitus tipo 2, distúrbios do sono, hipertensão arterial sistêmica, dislipidemia, lombalgias, osteoartroses, entre outros $;^{14} \mathrm{com} \mathrm{IMC} \geq 40 \mathrm{~kg} / \mathrm{m}^{2}$; que realizaram bypass gástrico em Y de Roux (técnica de Fobi-Capella) há no mínimo seis meses durante o período de coleta; nível de escolaridade mínima com ensino fundamental incompleto; e que anuíram ao TCLE apresentado no Apêndice A.

Foram excluídos da amostra indivíduos com idade inferior a 18 e superior a 65 anos; com IMC $<35 \mathrm{~kg} / \mathrm{m}^{2}$ (com ou sem comorbidades associadas); com pós-operatório tardio (a partir de 19 meses); que realizaram outra técnica cirúrgica excluindo o bypass gástrico em Y de Roux, que não anuíram ao TCLE e pacientes que não comparecerem à consulta previamente agendada.

A coleta de dados foi realizada mediante avaliação antropométrica e aplicação de questionário impresso, disponível no Apêndice B. Os pacientes foram convidados a participar da pesquisa no dia de consulta pós-operatória no ambulatório de nutrição, conforme previsto pelo protocolo de cirurgia bariátrica da unidade, que foi aplicada enquanto os pacientes esperavam ser chamados para a consulta. Os questionários foram aplicados de duas maneiras: a primeira parte com entrevista semiestruturada, preenchida pelo pesquisador, e uma segunda parte, com avaliação da qualidade de vida proposta pelo método BAROS, conforme o Anexo A, que foi preenchido pelo próprio pelo próprio paciente.

O questionário foi criado a partir da revisão bibliográfica, visando englobar: a) informações sociodemográficas, como sexo, idade, nível de escolaridade; b) morbidades antes e após a cirurgia; c) dados antropométricos ${ }^{11}$ referentes ao período pré-operatório, como peso pré-operatório, peso máximo atingido ao longo da vida, IMC pré-operatório, peso ideal, excesso de peso, percentual de perda de peso pré-operatório; d) dados antropométricos referentes ao pós-operatório, como peso atual, peso mínimo, IMC pós-operatório, percentual de perda de excesso de peso; e) hábitos de 
vida no pré e pós-operatório, incluindo etilismo, tabagismo e prática de atividade física (Apêndice B). Para mensurar a qualidade de vida, foi utilizado parcialmente o instrumento Bariatric Analysis and Reporting Outcome System (BAROS), ${ }^{11}$ adaptado do original para a língua portuguesa (Anexo A).

As comorbidades foram relatadas pelo próprio paciente durante a aplicação do questionário, sem consulta em prontuário ou análise de exames.

Em relação aos dados antropométricos, o peso pré-operatório foi consultado no prontuário do paciente, o peso máximo atingido ao longo da vida foi referido pelo paciente durante a entrevista, o peso atual pós-operatório foi aferido por meio de balança digital da marca Líder®, com capacidade de $300 \mathrm{~kg}$ e precisão de 100 gramas, enquanto que a medida da altura foi obtida por meio de estadiômetro acoplado a balança digital, com comprimento máximo de dois metros e escala de $0,5 \mathrm{~cm}$, estando o indivíduo descalço, em pé, com o mínimo de roupa, em posição ereta e braços relaxados ao longo do corpo, calcanhares unidos e olhar direcionado ao horizonte. Os demais dados (IMC pré-operatório, peso ideal, excesso de peso, percentual de perda de peso pré-operatório, IMC pós-operatório, \% percentual de perda de excesso de peso) foram calculados.

O percentual de perda de peso pré-operatório foi calculado por meio do peso máximo alcançado subtraído do peso pré-operatório, dividido pelo peso máximo e multiplicado por cem. Como peso ideal, considerou-se o valor médio de IMC de $24,9 \mathrm{~kg} / \mathrm{m}^{2}$, por ser o limítrofe entre estado eutrófico e sobrepeso ${ }^{5}$. O excesso de peso foi calculado em quilogramas, ao subtrair-se o peso ideal do peso pré-operatório. O percentual de excesso de peso perdido (PEP\%) foi calculado pela razão entre peso perdido e excesso de peso.

Os dados sociodemográficos e antropométricos foram investigados por meio de análises descritivas como frequência absoluta, percentual e desvio padrão. Para avaliar variáveis categóricas, foi aplicado o teste $t$ de Student pareado; teste não paramétrico de Wilcoxon Mann Whitney para amostras independentes; e teste não paramétrico de Kruskal Wallis para comparação entre três amostras independentes.

Para avaliação das questões do instrumento BAROS, utilizou-se apenas a parte sobre qualidade de vida, em que cada questão apresenta escala de Likert categórica para marcar as opções. O item "autoestima" totalizou até 1 (um) ponto (Muito pior sobre mim = -1; Pior sobre mim = $-0,5$; O mesmo $=0$; Melhor sobre $\mathrm{mim}=+0,5$; Muito melhor sobre mim $=+1$ ). Os outros itens: atividade física, relação social, desempenho no trabalho e interesse sexual, poderiam totalizar até 0,5 ponto para cada item (Muito menos $=-0,5$; Menos $=-0,25 ; \mathrm{O}$ mesmo $=0$; Mais $=+0,25$; Muito mais $=+0,5)$.

A avaliação final da qualidade de vida computada resultou em valor numérico entre -3 (menos três) e +3 (mais três). Assim, a qualidade de vida foi classificada como muito diminuída $(-3$ a $-2,25)$, diminuída (-2 a $-0,75)$, inalterada (-0,5 a 0,5$)$, melhorada $(0,75$ a 2$)$ ou muito melhorada $(2,25 \text { a } 3)^{7}$. 
Estabeleceu-se o nível de significância de $5 \%$ ( $\mathrm{p}<0,05)$, e os dados foram analisados por meio do programa SPSS (Statistical Package for The Social Sciences) versão 16.0.

A pesquisa foi aprovada pelo Comitê de Ética em Pesquisa com Seres Humanos do Hospital Geral de Goiânia (CAAE 56567116.9.0000.0035).

\section{APÊNDICE A-QUESTIONÁRIO PARA COLETA DE DADOS}

Hospital Geral de Goiânia

Trabalho: Avaliação do perfil nutricional e qualidade de vida após realização de cirurgia bariátrica

\begin{tabular}{|l|l|}
\hline \multicolumn{1}{|l|}{ 1. IDENTIFICAÇÃO } \\
\hline Sexo: $\square$ feminino $\square$ masculino & Data de nascimento: \\
\hline Grau de escolaridade: & $\square$ Ensino Fundamental completo \\
$\square$ Ensino Fundamental incompleto $\quad \square$ Ensino Médio completo \\
$\square$ Ensino Médio incompleto $\quad \square$ Ensino Superior completo \\
$\square$ Ensino Superior incompleto & $\square$ Hipertensão arterial (pressão alta) \\
\hline 2. DOENÇAS ASSOCIADAS & $\square$ Diabetes \\
$\square$ Hipertensão arterial (pressão alta) & $\square$ Dislipidemia \\
$\square$ Diabetes & (Ex: colesterol alto, triglicerídeo alto) \\
$\square$ Dislipidemia & $\square$ Osteoartrite \\
(Ex: colesterol alto, triglicerídeo alto) & $\square$ Osteoartrose \\
$\square$ Osteoartrite & $\square$ Lombalgia (dor na região lombar, \\
$\square$ Osteoartrose & $\square$ Costas) \\
$\square$ Lombalgia (dor na região lombar, & $\square$ Distúrbios do sono \\
costas) & $\square$ OUAS: \\
$\square$ Distúrbios do sono & $\square$ OUTRAS:
\end{tabular}




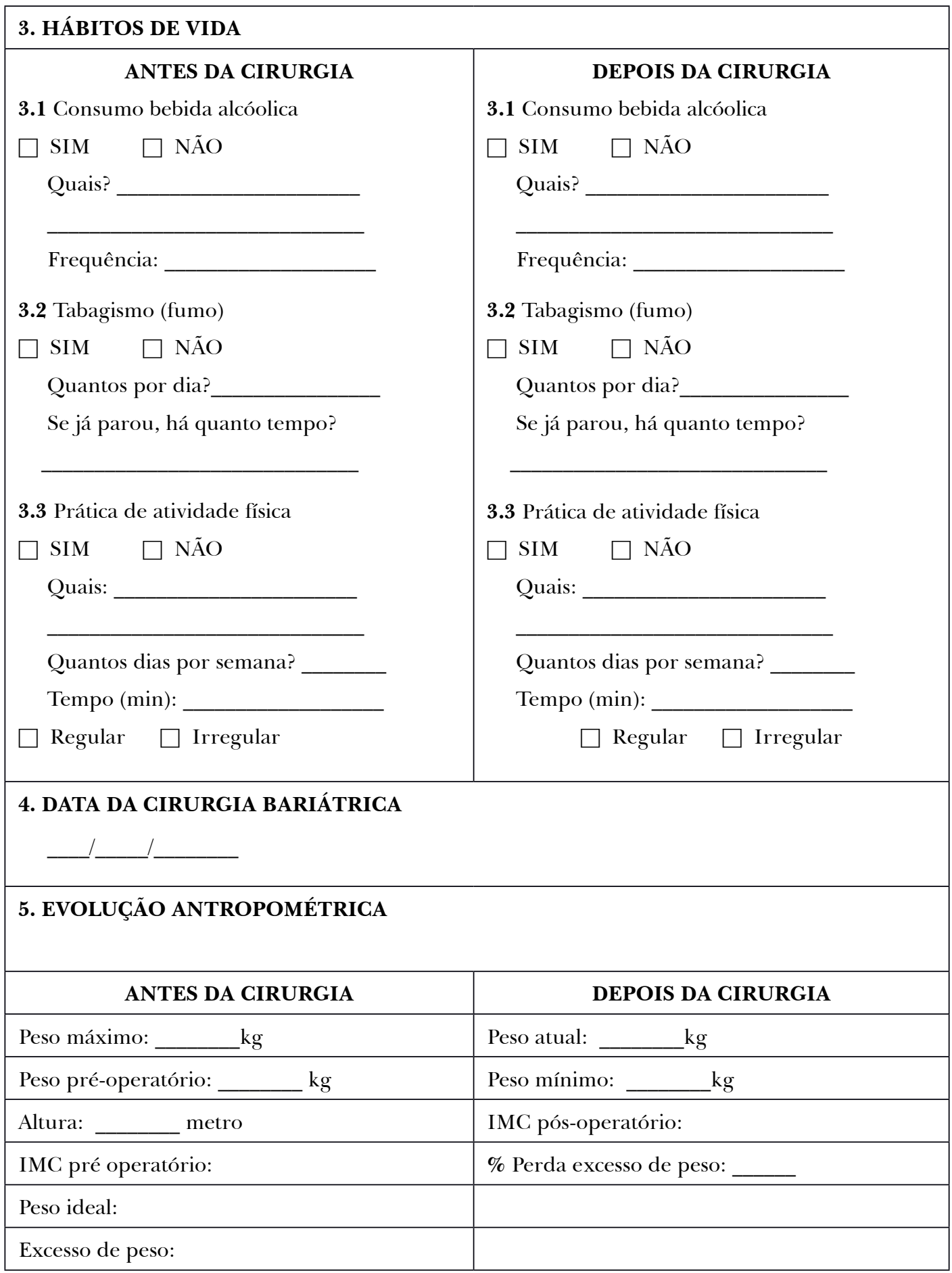


1. Comparado ao tempo antes de meu tratamento de perda de peso eu me sinto:

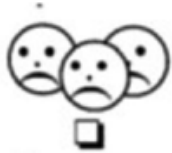

Muito pior sobre mim

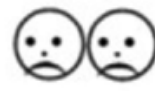

Pior sobre mim
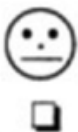

$\mathrm{O}$ mesmo

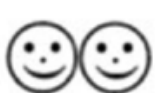

a

Melhor sobre mim

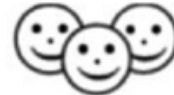

口

Muito melhor sobre mim

2. Eu sou capaz de participar fisicamente em atividades:

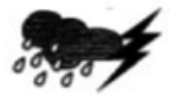

口

Muito menos

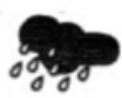

Menos
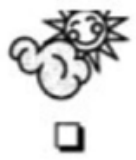

$\mathrm{O}$ mesmo
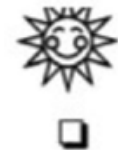

Mais
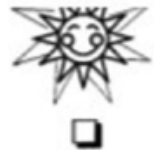

Muito mais

3. Estou disposto a me envolver socialmente:

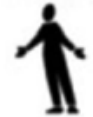

Muito menos

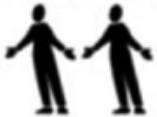

Menos

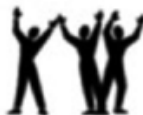

$\mathrm{O}$ mesmo

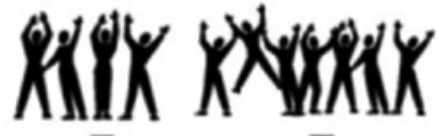

Mais

Muito mais

4. Eu sou capaz de trabalhar:

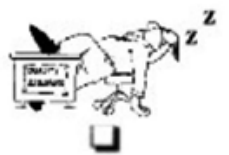

Muito menos

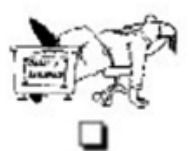

Menos

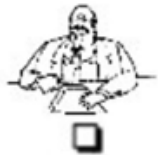

O mesmo

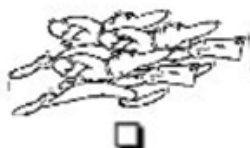

Mais

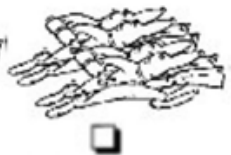

Muito mais

5. Estou interessado em sexo:

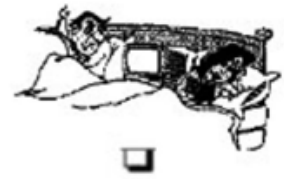

Muito menos

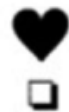

Menos

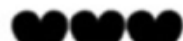

Mais

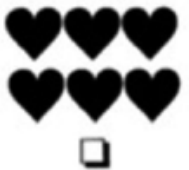

Muito mais 


\section{Resultados e discussão}

A amostra final foi composta por 56 pacientes em período pós-operatório de 6 a 18 meses de cirurgia bariátrica pela técnica de Fobi-Capella, com média de idade de 46 anos ( $\mathrm{DP}=9,61$ ), sendo $84 \%$ do sexo feminino $(n=4547)$ e $16 \%$ do masculino $(n=9)$. O número máximo de pacientes convidados possíveis de incluir na pesquisa era 77, e foram excluídas 21 pessoas $(29,9 \%)$, devido ao não comparecimento na consulta nutricional.

Em relação ao tempo de pós-operatório, o grupo 1 totalizou 20 pacientes (36\%); o grupo 2, 21 pacientes (37\%); e o grupo 3, 15 pacientes (27\%). As variáveis encontradas entre os grupos foram caracterizadas como independentes entre si e foram utilizadas para delinear o perfil antropométrico e a percepção da qualidade de vida em períodos pontuais de pós-cirúrgico.

$\mathrm{Na}$ amostra estudada, houve predomínio de indivíduos do sexo feminino, o que corrobora os trabalhos desenvolvidos por Rangel et al. ${ }^{16}$ e Oliveira et al. ${ }^{17}$, que encontraram prevalências de 76,6\% e 84,4\%, respectivamente, para as mulheres também submetidas a cirurgia bariátrica. Os dados epidemiológicos do Brasil e internacionais apontam que a obesidade afeta as pessoas independentemente do sexo; no entanto, tratando-se de realização de cirurgia bariátrica como tratamento da obesidade, observa-se maior frequência em mulheres.

Outro estudo conduzido nos EUA por Farinholt et al. ${ }^{18}$ apontou que mulheres são quatro vezes mais suscetíveis a procurar a cirurgia bariátrica do que os homens; a severidade e a frequência das complicações relacionadas ao excesso de peso são maiores nos homens. Apesar de o presente trabalho não ter como objetivo analisar a diferença entre sexo feminino e masculino, é importante investigar melhor a diferença de gênero na busca para o tratamento da obesidade e possíveis fatores associados.

A média de idade dos indivíduos desta pesquisa assemelhou-se ao estudo de Cunha et al., ${ }^{19}$ que encontraram média de 43,1 \pm 7,7 anos. Os níveis de escolaridade mais frequentes foram o ensino fundamental incompleto (46\%) e o ensino médio completo (23\%), dados similares ao estudo de Germano et al. ${ }^{20}$ realizado em João Pessoa, que analisaram o perfil nutricional de 51 pacientes de cirurgia bariátrica. Os autores encontraram que, dentre os níveis de escolaridade, também predominaram o ensino médio completo, seguido por ensino fundamental incompleto. A tabela 1 apresenta as características sociodemográficas como sexo, idade e escolaridade dos pacientes investigados. 
Tabela 1. Características sociodemográficas de indivíduos submetidos a cirurgia bariátrica em um hospital público de Goiânia-GO (2016).

\begin{tabular}{lc}
\hline \multicolumn{1}{c}{ Variável } & Amostra total \\
\hline Sexo $(\%)$ & $84 \%(\mathrm{n}=47)$ \\
Feminino & $16 \%(\mathrm{n}=9)$ \\
Masculino & $46 \pm 9,45$ \\
Idade (anos) & \\
Escolaridade & $46 \%(\mathrm{n}=26)$ \\
Ensino Fundamental Incompleto (\%) & $11 \%(\mathrm{n}=6)$ \\
Ensino Fundamental Completo $(\%)$ & $7 \%(\mathrm{n}=4)$ \\
Ensino Médio Incompleto (\%) & $23 \%(\mathrm{n}=13)$ \\
Ensino Médio Completo (\%) & $5 \%(\mathrm{n}=3)$ \\
Ensino Superior Incompleto $(\%)$ & $8 \%(\mathrm{n}=4)$ \\
Ensino Superior Completo (\%) &
\end{tabular}

A média de peso encontrada na amostra, considerando o período pré-operatório, foi de 131,1 $\pm 26,85 \mathrm{~kg}$, variando de 91 a $190 \mathrm{~kg}$. Os valores de IMC variaram entre 35,4 e 73,7 kg/m², com média de 50,1 $\pm 8,37 \mathrm{~kg} / \mathrm{m}^{2}$, corroborando dados apontados por Cunha et al., ${ }^{19}$ que encontraram média de IMC pré-operatório de $51,2 \mathrm{~kg} / \mathrm{m}^{2}\left(40,2-74,1 \mathrm{~kg} / \mathrm{m}^{2}\right)$.

A perda de peso antes da cirurgia foi de 8,7\% (DP 6,5), dado que concorda com a estimativa de perda de $10 \%$ do peso máximo, estabelecido pelo protocolo da unidade hospitalar analisada e que também se enquadra nas faixas de recomendação para um melhor preparo e maiores chances de sucesso. A perda de peso pré-operatória deve ser incentivada por propiciar redução das comorbidades associadas à obesidade, diminuição do risco de complicações associadas à cirurgia e melhores resultados pós-operatórios. ${ }^{21}$

A perda de peso no período pré-operatório apresentou diferença significativa entre os grupos $(\mathrm{p}=0,014)$ e foi maior nos pacientes com 18 meses de pós-operatório. A hipótese sugerida para essa diferença pode ser explicada pelo fato de que esse grupo apresentou valores mais elevados de peso máximo alcançado ao longo da vida (média 161,2kg) e peso antes da cirurgia (média $141,6 \mathrm{~kg}$ ) em relação aos grupos de seis e 12 meses, sendo estimulados a perder o máximo possível de peso até o momento da cirurgia. 
Após a realização da cirurgia bariátrica, a média de peso encontrado foi de $92,6 \mathrm{~kg} \pm 18,63$, e o IMC pós-operatório foi de 35,5 kg/m² $\left(24,92-60,66 \mathrm{~kg} / \mathrm{m}^{2}\right)$. Pouco mais da metade da amostra (55\%) alcançou, até o momento da coleta, IMC superior a $25 \mathrm{~kg} / \mathrm{m}^{2}$ e inferior a $35 \mathrm{~kg} / \mathrm{m}^{2}$, classificado pela OMS como obesidade grau I. Esse dado é considerado positivo, uma vez que destaca a redução do grau de obesidade e a maior proximidade com o peso ideal.

A partir do dado "excesso de peso", foi calculado o percentual de perda de excesso de peso em relação ao peso pré-operatório, obtendo-se a média de perda de 60,4\% $\pm 15,71$ e maior perda no grupo de 12 meses pós-operatório, equivalente a 66,15\% ( $\mathrm{p}=0,005)$. Observou-se que grande parte dos participantes de 6 a 18 meses de pós-operatório (79\%) alcançou PEP acima de 50\%, parâmetro importante para definição de sucesso da cirurgia. O percentual de perda de excesso de peso de pelo menos $50 \%$ com a manutenção aos longos dos anos é uma das referências para interpretar o indicador de sucesso. ${ }^{22} \mathrm{~A}$ tabela 2 apresenta os dados antropométricos dos indivíduos investigados.

Tabela 2. Características antropométricas de indivíduos submetidos a cirurgia bariátrica em um hospital público de Goiânia-GO (2016).

\begin{tabular}{lccccc}
\hline \multicolumn{1}{c}{ Variável } & Amostra total & $6 \mathrm{~m} \mathrm{PO}$ & $12 \mathrm{~m} \mathrm{PO}$ & $18 \mathrm{~m} \mathrm{PO}$ & p-value $^{\mathrm{a}}$ \\
\hline Peso máximo $(\mathrm{kg})$ & $144,4 \pm 32,29$ & $144,1 \pm 30,73$ & $135,33 \pm 22,83$ & $161,2 \pm 40,35$ & 0,135 \\
Peso pré-operatório $(\mathrm{kg})$ & $131,1 \pm 26,85$ & $130,8 \pm 23,48$ & $126 \pm 22,27$ & $141,6 \pm 34,83$ & 0,471 \\
IMC pré-operatório $\left(\mathrm{kg} / \mathrm{m}^{2}\right)$ & $50,1 \pm 8,37$ & $49,9 \pm 9,07$ & $49,046,07$ & $52,6 \pm 9,95$ & 0,661 \\
Perda de peso pré-operatório $(\%)$ & $8,7 \pm 6,5$ & $8,3 \pm 8,43$ & $6,93 \pm 4,16$ & $11,9 \pm 4,68$ & 0,014 \\
Peso ideal $(\mathrm{kg})$ & $65,3 \pm 7,48$ & $65,8 \pm 6,73$ & $64,03 \pm 6,26$ & $67,1 \pm 9,74$ & 0,639 \\
Excesso de peso $(\mathrm{kg})$ & $65,9 \pm 23,06$ & $65 \pm 22,07$ & $61,97 \pm 18,14$ & $75,5 \pm 28,99$ & 0,506 \\
Peso pós-operatório $(\mathrm{kg})$ & $92,6 \pm 18,63$ & $100,1 \pm 20,22$ & $85,48 \pm 12,94$ & $93,8 \pm 19,72$ & 0,067 \\
IMC pós-operatório $\left(\mathrm{kg} / \mathrm{m}^{2}\right)$ & $35,5 \pm 6,99$ & $38,3 \pm 8,54$ & $33,35 \pm 3,71$ & $35,3 \pm 7,10$ & 0,180 \\
\% Perda excesso de peso & $60,4 \pm 15,71$ & $51,1 \pm 15,03$ & $66,15 \pm 12,86$ & $65,8 \pm 12,86$ & 0,005 \\
\hline
\end{tabular}

a. Teste $T$ para amostras independentes

b. $\mathrm{PO}=$ pós-operatório

A obesidade relaciona-se ao aumento do risco de desenvolver outros tipos de doenças crônicas associadas e maiores índices de mortalidade. ${ }^{4}$ Segundo relato dos entrevistados, a maioria apresentava uma ou mais comorbidades associadas (96,4\%) e apenas dois indivíduos $(3,6 \%)$ referiram não possuir nenhuma doença antes da cirurgia. As mais frequentes foram HAS (84\%), DM tipo $2(64 \%)$ e dislipidemias $(59 \%)(\mathrm{p}<0,001)$. 
No estudo realizado por Barros et al. ${ }^{23}$, também pesquisando condições clínicas de 92 pacientes submetidos a cirurgia bariátrica em Fortaleza-CE, a HAS se destacou como doença mais prevalente (50\%), seguida por DM2 e dislipidemias, ambas com frequência de $13 \%(\mathrm{p}=0,001)$. Similarmente, Costa et al. ${ }^{24}$ e Diniz et al..$^{25}$ encontraram a HAS como a comorbidade de maior prevalência entre as amostras investigadas, com valores de $63,49 \%$ e $63,2 \%$, respectivamente. O perfil investigado salienta a alta prevalência de doenças associadas à obesidade, como apontado na literatura, e altas taxas de resolução ainda nos primeiros anos de realização da cirurgia, devido à perda ponderal.

O percentual de melhora das comorbidades após a realização da cirurgia foi satisfatório e com diferença significativa $(\mathrm{p}<0,001)$. Os melhores resultados envolveram a resolução de distúrbios do sono (94\%), dislipidemias (91\%) e diabetes (86\%). O menor percentual de melhora foi para osteoartrose (43\%), provavelmente devido à menor possibilidade de reversão dessa doença, mesmo com a evolução positiva da perda de peso. A tabela 3 contém os dados das comorbidades antes e após a cirurgia bariátrica relatadas pelos pacientes analisados.

Em 2015, foi realizada pesquisa para avaliar o impacto da cirurgia bariátrica na qualidade de vida em pacientes no Estado de Goiás, estudo com objetivos semelhantes aos da presente análise. As comorbidades pré-operatórias mais prevalentes entre o público-alvo foram HAS, dislipidemias e diabetes, apresentando boas taxas de resolução após a realização da cirurgia bariátrica - 77\%, $100 \%$ e $100 \%$, respectivamente - dados que se encontram similares aos achados atuais. ${ }^{26}$

Tabela 3. Comorbidades antes e depois da cirurgia a autorrelatadas por indivíduos submetidos a cirurgia bariátrica em um hospital público de Goiânia-GO (2016).

\begin{tabular}{|c|c|c|c|c|c|c|c|}
\hline \multirow[t]{2}{*}{ Variável } & \multicolumn{2}{|c|}{$\begin{array}{c}\text { Comorbidades antes } \\
\text { da cirurgia } \\
\mathrm{N}(\%)\end{array}$} & \multicolumn{2}{|c|}{$\begin{array}{c}\text { Comorbidades } \\
\text { depois da cirurgia } \\
\mathrm{N}(\%)\end{array}$} & \multirow[t]{2}{*}{$\mathrm{N}$ total } & \multirow[t]{2}{*}{$\begin{array}{c}\text { Melhora das } \\
\text { comorbidades } \\
(\%)\end{array}$} & \multirow[t]{2}{*}{$p$-value ${ }^{b}$} \\
\hline & Sim & Não & Sim & Não & & & \\
\hline HAS & $47(84 \%)$ & $9(16 \%)$ & $18(32 \%)$ & $38(68 \%)$ & 56 & 62 & $<0,001$ \\
\hline DM 2 & $36(64 \%)$ & $20(36 \%)$ & $5(9 \%)$ & $51(91 \%)$ & 56 & 86 & $<0,001$ \\
\hline Dislipidemias & $33(59 \%)$ & $23(41 \%)$ & $3(5 \%)$ & $53(95 \%)$ & 56 & 91 & $<0,001$ \\
\hline Osteoartrose & $28(50 \%)$ & $28(50 \%)$ & $16(29 \%)$ & $40(7 \%)$ & 56 & 43 & $<0,001$ \\
\hline Lombalgia & $31(55 \%)$ & $25(45 \%)$ & $11(20 \%)$ & $45(80 \%)$ & 56 & 65 & $<0,001$ \\
\hline Distúrbios do sono & $18(32 \%)$ & $38(68 \%)$ & $1(2 \%)$ & $55(98 \%)$ & 56 & 94 & $<0,001$ \\
\hline
\end{tabular}

a. Valores constituem número total e percentual.

b. Teste pareado de $\mathrm{T}$ de student. 
Para a variável "hábitos de vida”, foram mensurados consumo de bebida alcóolica, tabagismo e prática de atividade física. Dos pacientes entrevistados, $32 \%(n=18)$ referiram consumo eventual de bebida alcóolica antes da realização da cirurgia bariátrica, e as bebidas consumidas foram as do tipo fermentadas, como cerveja e vinho.

Após a realização de cirurgia, apenas 3,7\% dos indivíduos referiram ingerir bebidas alcóolicas eventualmente. Esses resultados assemelham-se ao estudo realizado em Recife, por Amorim et al. ${ }^{27}$, sobre uso de bebida alcóolica no período pré e pós-operatório de cirurgia bariátrica. Esses autores encontraram que 26,6\% dos pacientes ingeriam bebida alcóolica no período pré-operatório, predominando o consumo de cerveja. Infere-se que a redução no consumo de bebida alcóolica observada após a cirurgia possa ser relacionada à efetividade das orientações nutricionais da equipe multiprofissional realizadas na unidade hospitalar onde o presente estudo foi realizado, por meio das quais se desestimula o consumo de bebida alcoólica de até, no mínimo, 12 meses de cirurgia. Além disso, sugere-se que possa ter ocorrido sub-relato de informações.

Em relação ao tabagismo, observou-se que 25\% (n=14) da amostra eram ex-tabagistas antes da realização da cirurgia. O período de cessação de fumo foi variado de um ano a 27 anos, com média de 13 anos. No período pós-operatório, todos os pacientes relataram não fumar. O tabagismo configura sério fator de risco para o equilíbrio no estado de saúde e é considerado um hábito não saudável. Recomenda-se no protocolo da unidade a cessação do tabagismo pelo menos dois meses antes da cirurgia, dado que foi ao encontro do esperado.

Antes da cirurgia bariátrica, $59 \%$ das pessoas referiram sedentarismo e $41 \%(n=23)$ relataram praticar atividade física, sendo a modalidade mais citada a caminhada. Depois da cirurgia, 40 pacientes $(71 \%)$ referiram praticar atividade física. Das 23 pessoas que realizavam prática de atividade física antes, todas referiram permanecer com o hábito $(\mathrm{p}=0,001)$. A prática de atividade física é um hábito componente do estilo de saudável, importante para perda de peso e manutenção ao longo do tempo. O sedentarismo identificado no período pré-operatório pode estar relacionado à limitação funcional provocada pelo excesso de peso, presença de determinadas comorbidades, falta de tempo, acesso físico e financeiro, autoestima, entre outros.

A avaliação da qualidade de vida ocorreu por meio do instrumento BAROS, com cinco domínios: autoestima, disposição para atividade física, desempenho no trabalho, interação social e prática de atividade sexual. Da amostra total, $27 \%$ dos indivíduos avaliaram sua qualidade de vida como melhorada e 73\% como muito melhorada. Nenhum indivíduo considerou sua qualidade de vida como diminuída, muito diminuída ou inalterada após realização da cirurgia bariátrica, dados disponíveis na tabela 4.

Analisando-se os três períodos pós-operatórios e os indivíduos que tiveram perda ponderal inferior e superior a $50 \%$, não foram constatadas diferenças significativas, Vale destacar, no entanto, que desde os seis meses pós-operatório os pacientes já indicaram melhora na percepção 
da sua qualidade de vida. A tabela 5 apresenta relação entre perda do excesso de peso e percepção de qualidade de vida. Em outra investigação realizada no Estado de Goiás, $8 \%$ dos pacientes relataram se sentir melhor após a cirurgia e $92 \%$ muito melhor, corroborando o perfil encontrado neste estudo. ${ }^{26}$ Infere-se que a perda ponderal alcançada nos primeiros meses e ainda antes da estabilização do peso já propicia melhoras na qualidade de vida, não sendo necessariamente associada a melhor percepção, conforme maior perda de peso.

Tabela 4. Percepção da qualidade de vida por indivíduos submetidos a cirurgia bariátrica em um hospital público de Goiânia-GO proposto pelo método BAROSa (2016).

\begin{tabular}{lccccc}
\hline $\begin{array}{c}\text { Variável qualidade } \\
\text { de vida }\end{array}$ & $\begin{array}{c}\text { Amostra total } \\
\%(\mathrm{n})\end{array}$ & $\begin{array}{c}\text { Grupo 1 } \\
\%(\mathrm{n})\end{array}$ & $\begin{array}{c}\text { Grupo } 2 \\
\%(\mathrm{n})\end{array}$ & $\begin{array}{c}\text { Grupo 3 } \\
\%(\mathrm{n})\end{array}$ & p-value $^{\mathrm{b}}$ \\
\hline $\begin{array}{l}\text { Muito diminuída } \\
\text { Diminuída }\end{array}$ & - & - & - & - & - \\
Inalterada & - & - & - & - & - \\
Melhorada & - & - & - & - & - \\
Muito melhorada & $27 \%(15)$ & $20 \%(4)$ & $38 \%(8)$ & $20 \%(3)$ & 0,261 \\
\hline
\end{tabular}

a. BAROS (Bariatric Analysis and Reporting Outcome System).

b. Teste Kruskal-Wallis.

c. Grupo 1 = 6 meses pós-operatório; Grupo 2 = 12 meses pós-operatório e Grupo 3 = 18 meses pós-operatório.

Tabela 5. Relação entre perda do excesso de peso e percepção de qualidade de vida ${ }^{a}$ de indivíduos submetidos a cirurgia bariátrica em um hospital público de Goiânia-GO (2016).

\begin{tabular}{lccc}
\hline \multicolumn{1}{c}{ Variável $^{\mathrm{b}}$} & $\begin{array}{c}\% \mathrm{PEP}^{\mathrm{c}}<50 \\
(\mathrm{n}=12)\end{array}$ & $\begin{array}{c}\% \text { PEP }>50 \\
(\mathrm{n}=44)\end{array}$ & p-value $^{\mathrm{d}}$ \\
\hline Autoestima & $0,88 \pm 0,23$ & $0,95 \pm 0,15$ & 0,163 \\
Disposição para atividade física & $0,38 \pm 0,17$ & $0,43 \pm 0,12$ & 0,273 \\
Desempenho no trabalho & $0,40 \pm 0,17$ & $0,39 \pm 0,20$ & 0,929 \\
Interação social & $0,33 \pm 0,19$ & $0,33 \pm 0,22$ & 0,963 \\
Prática de atividade sexual & $0,33 \pm 0,19$ & $0,21 \pm 0,28$ & 0,188 \\
Classificação final & $2,31 \pm 0,64$ & $2,29 \pm 0,66$ & 0,975 \\
\hline
\end{tabular}

a. Valores constituem média \pm desvio-padrão

b. Média por variável: autoestima (0-1); disposição para atividade física, desempenho no trabalho, interação social e prática de atividade sexual (0-0,5); classificação final (0-3).

c. $\mathrm{PEP}=$ Perda de excesso de peso

d. Teste não paramétrico de Wilcoxon Mann Whitney 
O instrumento BAROS, contudo, não foi utilizado na pesquisa em sua totalidade para avaliar o resultado/sucesso da cirurgia bariátrica devido a limitações do serviço de saúde analisado, como falta de padronização do registro de complicações no período pós-operatório e reavaliações das comorbidades. O BAROS é considerado o modo mais eficaz direcionado à avaliação global do tratamento cirúrgico da obesidade. No entanto, inúmeras críticas têm sido elencadas por pesquisadores, devido a limitações no seu desenho, como a impossibilidade de comparar o estado de saúde do pré com o pós-operatório, valores expressos em categorias nominais para cada domínio agrupando o mesmo modo indivíduos com características e resultados diferentes, limitação das comorbidades listadas para serem avaliadas, entre outros. ${ }^{28}$

Os resultados alcançados reforçam a importância da perda de peso para redução dos fatores de risco, melhora de doenças associadas, melhora na qualidade de vida e na mortalidade. Uma das limitações do estudo foi o desenho transversal, ao utilizar uma única análise para caracterização de um aspecto, o que dificulta a realização de associações e comparações. Para investigações futuras, indica-se a elaboração de estudos prospectivos, com acompanhamento de um único grupo ao longo do tempo, com uso ou não de caso controle, de modo que as mudanças antropométricas, nos hábitos e na qualidade de vida possam ser investigadas com mais detalhes e maiores associações.

A reformulação do BAROS ou criação de outro instrumento apropriado para avaliar os resultados do tratamento cirúrgico da obesidade é importante para análises mais fidedignas, maior padronização nas coletas e comparações entre diferentes estudos.

Além disso, outros pontos relevantes que podem ser associados em outros estudos são a caracterização do perfil alimentar antes e depois da cirurgia, uso de suplementação e exames bioquímicos, taxa metabólica basal, gasto energético total. Assim, as interpretações podem ter maior embasamento e ser relacionadas com a perda ponderal, adoção ou retirada de hábitos e melhora na qualidade de vida.

\section{Conclusão}

O estudo apontou que a realização da cirurgia bariátrica tem propiciado alta perda de peso, melhora e/ou resolução de comorbidades associadas e melhora na qualidade de vida antes da estabilização da perda de peso. Constatou-se também a presença de hábitos saudáveis e protetores para saúde, como baixa prevalência no consumo de bebidas alcoólicas, ausência de tabagismo e prática de atividade física. Foram alcançados os objetivos propostos, e a amostra estudada salienta a eficácia da cirurgia bariátrica no tratamento da obesidade em casos de refratariedade no tratamento convencional e conforme os critérios de indicação. Todavia, destaca-se a importância de avaliar o sucesso da cirurgia com maior tempo de pós-operatório, visando observar a manutenção 
ou reganho do peso, bom controle e/ou resolução de comorbidades, presença de deficiências nutricionais e hábitos de vida.

Sugere-se a realização de outras pesquisas que investiguem o conceito qualidade de vida e seus domínios e o perfil nutricional e alimentar dos pacientes submetidos a cirurgia bariátrica.

\section{Colaboradores}

Maia RP contribuiu na concepção do estudo, coleta de dados, análise e interpretação dos dados e redação do manuscrito; da Silva PCC contribuiu na concepção do estudo e coleta de dados; Duarte ACS participou da orientação e revisão do manuscrito; da Costa RM participou da coorientação do estudo e revisão do manuscrito.

Conflito de interesses: Os autores declaram não haver conflitos de interesses.

\section{Referências}

1. Cuppari L. Nutrição: nas doenças crônicas não-transmissíveis. $3^{\text {a }}$ ed. Barueri, SP: Manole; 2009.

2. Associação Brasileira para o Estudo da Obesidade e da Síndrome Metabólica (Abeso). Diretrizes Brasileiras Obesidade 2009/2010. 3.ed. Itapevi: AC Farmacêutica; 2009.

3. Mancini MC. Obesidade e Doenças Associadas. In: Mancini MC, Geloneze B, Salles JEN, Lima JG, Carra MK. Tratado de Obesidade. Itapevi: AC Farmacêutica 2010, p. 253-264.

4. Ministério da Saúde. Secretaria de Vigilância em Saúde. Departamento de Vigilância de Doenças e Agravos não Transmissíveis e Promoção da Saúde. Vigitel Brasil 2014: vigilância de fatores de risco e proteção para doenças crônicas por inquérito telefônico. Brasília: Ministério da Saúde; 2015: 152p.

5. World Health Organization. Obesity: preventing and managing he global epidemic. Report of a World Health Organization Consultation. Geneva: World Health Organization; 2000; n. 284: p. 256.

6. Garrido Jr, AB. Cirurgia da obesidade. Rio de Janeiro: Atheneu; 2003: 228p.

7. Wolfe BM, Kvach E, Eckel RH. Treatment of Obesity: Weight Loss and Bariatric Surgery Circ Res 2016; 118(11): 1844-1855.

8. Pereira EF, Teixeira CS, Santos A. Qualidade de vida: abordagens, conceitos e avaliação. Rev bras Educ Fís Esporte, São Paulo, 2012; 26(2): 241-250.

9. Dare S, Mackay DF, Pell JP. Relationship between Smoking and Obesity: A Cross-Sectional Study of 499,504 Middle Aged Adults in the UK General Population. PLoS ONE 2015; 10(4): e0123579.

10. Andersen LL, Izquierdo M, S Emil. Overweight and obesity are progressively associated with lower work ability in the general working population: cross-sectional study among 10,000 adults. Int Arch Occup Environ Health 2017; 90: 779-787. 
11. Oria HE, Moorehead MK. Bariatric analysis and reporting outcome system (BAROS). Obes Surg 1998 Oct; 8(5): 487-499.

12. Moraes JM, Caregnato RCA, Schneider DS. Qualidade de vida antes e após a cirurgia bariátrica. Acta Paul Enferm 2014; 27(2): 157-64.

13. Barros LM, Moreira RAN, Frota NM, Caetano JA. Mudanças na qualidade de vida após a cirurgia bariátrica. Ver Enferm UFPE 2013; 7(5): 1265-75.

14. Conselho Federal de Medicina. Resolução CFM N 1942/2010. Altera a Resolução CFM no 1.766, de 13 de maio de 2005, publicada no Diário Oficial da União em 11 de julho de 2005, Seção I, página 114, que estabelece normas seguras para o tratamento cirúrgico da obesidade mórbida, definindo indicações, procedimentos e equipe. Diário Oficial da União 12 fev 2010; Seção 1: p.72.

15. Mussoi TD. Avaliação Nutricional na Prática Clínica - da Gestação ao Envelhecimento. [S.I]: Guanabara Koogan; 2014.328p.

16. Rangel LOB, Faria VSP, Magalhães EA, Araújo ACT, Bastos EMRD. Perfil de saúde e nutricional de pacientes portadores de obesidade mórbida candidatos à cirurgia bariátrica. Rev Bras Nutr Clin 2007; 22(3): 214-9.

17. Oliveira APF, Malheiros CA, Santos AS, Jesus SR, Manuel J. Perfil de pacientes submetidos à cirurgia bariátrica atendidos em um hospital universitário do município de São Paulo. Saúde Coletiva 2009; 06(35): 275-279.

18. Farinholt GN, Carr AD, Chang EJ, Ali MR. A call to arms: obese men with more severe comorbid disease and under utilization of bariatric operations. Surg Endosc 2013; 27: 4556-4563.

19. Cunha SFC, Sanches M, Faria A, Santos JE, Nonino-Borges CB. Evolução da massa corporal magra após 12 meses da cirurgia bariátrica. Rev. Nutr 2010; 23(4): 535-541.

20. Germano ACPL, Camelo CMBM, Batista FM, Carvalho NMA, Liberali R, Coutinho VF. Perfil nutricional dos pacientes submetidos à cirurgia bariátrica e dos critérios adotados para encaminhamento em um Hospital De João Pessoa, PB. Ensaios e Ciência: Ciências Biológicas, Agrárias e da Saúde 2010; 14(2): 43-59.

21. Santos LG, Araújo MSM. Perda de peso pré-operatória em pacientes submetidos à gastroplastia redutora com derivação gastrointestinal em y-de-roux: uma revisão de literatura. Com Ciências Saúde 2012; 24(2): 127-134.

22. Fobi MA. Surgical treatment of obesity: a review. J Natl Med Assoc 2004; 96(1): 61-75.

23. Barros LM, Frota NM, Moreira RAN, Araújo TM, Caetano JA. Avaliação dos resultados da cirurgia bariátrica. Rev Gaúcha Enferm 2015; 36(1): 21-27.

24. Costa ACC, Ivo ML, Cantero WB, Tognini JRF. Obesidade em pacientes candidatos a cirurgia bariátrica. Acta Paul Enferm 2009; 22(1): 55-59.

25. Diniz MFHS, Passos VMA, Barreto SM, Diniz MTC, Linares DB, Mendes LN. Perfil do paciente obeso classe III do Sistema Público de Saúde submetidos à gastroplastia em "Y de Roux", no Hospital das clínicas da UFMG: altas prevalências de superobesidade, comorbidades e mortalidade hospitalar. Rev Med Minas Gerais 2008; 18(3): 183-190. 
26. Ribeiro EM, Ávila RI, Santos RRS, Garrote CFD. Impact of bariatric surgery on patients from Goiás, Brazil, using the BAROS method - a preliminary study. GE Port J Gastroenterol 2015; 22(3): 93-102.

27. Amorim ACR, Souza AFO, Nascimento ALV, Maio R, Burgos MGPA. Uso de bebida alcoólica em períodos pré e pós-operatório de cirurgia bariátrica. Rev Col Bras Cir 2015; 42(1): 003-008.

28. Nicareta JR, Freitas ACT, Nicareta SM, Nicareta C, Campos ACL, Nassif PAN, et al. Análise crítica do método BAROS (Bariatric Analysis and Reporting System). ABCD Arq Bras Cir Dig 2015; 28(1): 73-78.

Recebido: 22 de fevereiro, 2017

Revisado: 06 de agosto, 2017

Aceito: 12 de janeiro, 2018 